\title{
Recent results in nuclear astrophysics at the n_TOF facility at CERN
}

\section{G. Tagliente ${ }^{1}$}

Istiruto Nazionale di Fisica Nucleare, Sezione di Bari, Italy

Via Orabona 4, I-70125 Bari Italy

E-mail: giuseppe. tagliente@ba.infn. it

O. Aberle $^{8}$, J. Andrzejewski ${ }^{7}$, L. Audouin ${ }^{16}$, M. Bacak $^{11,8,12}$, J. Balibrea ${ }^{6}$, M. Barbagallo $^{1}$, F. Bečváŕ ${ }^{19}$, E. Berthoumieux ${ }^{12}$, J. Billowes ${ }^{17}$, D. Bosnar ${ }^{18}$, A. Brown $^{20}$, M. Caamaño ${ }^{4}$, F. Calviño ${ }^{21}$, M. Calviani ${ }^{8}$, D. Cano-Ott ${ }^{6}$, R. Cardella ${ }^{8}$, A. Casanovas $^{21}$, F. Cerutti ${ }^{8}$, Y. H. Chen ${ }^{16}$, E. Chiaveri ${ }^{8,17,2}$, N. Colonna ${ }^{1}$, G. Cortés ${ }^{21}$, M. A. Cortés-Giraldo ${ }^{2}$, Cosentino ${ }^{22}$, L. A. Damone ${ }^{1,23}$, M. Diakaki ${ }^{12}$, C. DomingoPardo $^{3}$, L. R. Dressler ${ }^{14}$, E. Dupont ${ }^{12}$, I. Durán ${ }^{4}$, B. Fernández-Domínguez ${ }^{4}$, A. Ferrari $^{8}$, P. Ferreira ${ }^{10}$, P. Finocchiaro ${ }^{22}$, V. Furman ${ }^{24}$, K. Göbel ${ }^{5}$, A. R. García ${ }^{6}$, A. Gawlik $^{7}$, S. Gilardoni ${ }^{8}$, T. Glodariu ${ }^{9}$, I. F. Gonçalves ${ }^{10}$, E. González $^{6}$, E. Griesmayer $^{11}$, C. Guerrero ${ }^{2}$, F. Gunsing ${ }^{12,8}$, H. Harada ${ }^{25}$, S. Heinitz ${ }^{14}$, J. Heyse ${ }^{26}$, D. G. Jenkins ${ }^{20}$, E. Jericha ${ }^{11}$, F. Käppeler ${ }^{27}$, Y. Kadi ${ }^{8}$, A. Kalamara ${ }^{28}$, P. Kavrigin ${ }^{11}$, A. Kimura $^{25}$, N. Kivel ${ }^{14}$, M. Kokkoris ${ }^{28}$, M. Krtička ${ }^{19}$, D. Kurtulgil ${ }^{5}$, E. Leal-Cidoncha ${ }^{4}$, C. Lederer ${ }^{29}$,J. Lerendegui-Marco ${ }^{2}$, H. Leeb ${ }^{11}$, S. Lo Meo ${ }^{15,30}$, S. J. Lonsdale ${ }^{29}$, D. Macina $^{8}$, J. Marganiec ${ }^{7,13}$, T. Martínez ${ }^{6}$, A. Masi ${ }^{8}$, C. Massimi ${ }^{30,31}$, P. Mastinu ${ }^{32}$, M. Mastromarco $^{1}$, E. A. Maugeri ${ }^{14}$, A. Mazzone ${ }^{1,33}$, E. Mendoza ${ }^{6}$, A. Mengoni ${ }^{15}$, F. Mingrone $^{8}$, P. M. Milazzo ${ }^{34}$, A. Musumarra ${ }^{22,35}$, A. Negret ${ }^{9}$, R. Nolte ${ }^{13}$, A. Oprea ${ }^{9}$, N. Patronis ${ }^{36}$, A. Pavlik ${ }^{37}$, J. Perkowski ${ }^{7}$, I. Porras ${ }^{38}$, J. Praena ${ }^{38}$, J. M. Quesada ${ }^{2}$, D. Radeck ${ }^{13}$, T. Rauscher ${ }^{39,40}$, R. Reifarth ${ }^{5}$, C. Rubbia ${ }^{8}$, J. A. Ryan ${ }^{17}$ M. SabatéGilarte $^{8,2}$, A. Saxena ${ }^{41}$, P. Schillebeeckx ${ }^{26}$, D. Schumann ${ }^{14}$, P. Sedyshev ${ }^{24}$, A. G. Smith $^{17}$, N. V. Sosnin ${ }^{17}$, A. Stamatopoulos ${ }^{28}$, J. L. Tain ${ }^{3}$, A. Tarifeño-Saldivia ${ }^{21}$ L. Tassan-Got $^{16}$, S. Valenta ${ }^{19}$, G. Vannini ${ }^{30,31}$, V. Variale ${ }^{1}$, P. Vaz $^{10}$, A. Ventura ${ }^{30}$, V. Vlachoudis $^{8}$, R. Vlastou ${ }^{28}$, A. Wallner ${ }^{42}$, S. Warren ${ }^{17}$, P. J. Woods ${ }^{29}$ T. Wright ${ }^{17}$, P. Čżugec ${ }^{18,8}$

and the n_TOF Collaboration

${ }^{1}$ Istituto Nazionale di Fisica Nucleare, Sezione di Bari, Italy ${ }^{2}$ Universidad de Sevilla, Spain, ${ }^{3}$ Instituto de Física Corpuscular, Universidad de Valencia, Spain, ${ }^{4}$ University of Santiago de Compostela, Spain ${ }^{5}$ Goethe University Frankfurt, Germany, ${ }^{6}$ Centro de Investigaciones Energéticas Medioambientales y Tecnológicas (CIEMAT), Spain, ${ }^{7}$ University of Lodz, Poland, ${ }^{8}$ European Organization for Nuclear Research (CERN), Switzerland, ${ }^{9}$ Horia Hulubei National Institute of Physics and Nuclear Engineering, Romania, ${ }^{10}$ Instituto Superior Técnico, Lisbon, Portugal, ${ }^{11}$ Technische Universität Wien, Austria, ${ }^{12} \mathrm{CEA}$ Saclay, Irfu, Gif-sur-Yvette, France, ${ }^{13}$ Physikalisch-Technische Bundesanstalt (PTB), Bundesallee 100, 38116 Braunschweig, Germany, ${ }^{14}$ Paul Scherrer Institut (PSI), Villingen, Switzerland, ${ }^{15}$ Agenzia

$1 \quad$ Speaker 
nazionale per le nuove tecnologie (ENEA), Bologna, Italy, ${ }^{16}$ Institut de Physique Nucléaire, CNRSIN2P3, Univ. Paris-Sud, Université Paris-Saclay, F-91406 Orsay Cedex, France, ${ }^{17}$ University of Manchester, United Kingdom, ${ }^{18}$ University of Zagreb, Croatia, ${ }^{19}$ Charles University, Prague, Czech Republic, ${ }^{20}$ University of York, United Kingdom, ${ }^{21}$ Universitat Politècnica de Catalunya, Spain, ${ }^{22}$ INFN Laboratori Nazionali del Sud, Catania, Italy, ${ }^{23}$ Dipartimento di Fisica, Università degli Studi di Bari, Italy, ${ }^{24}$ Joint Institute for Nuclear Research (JINR), Dubna, Russia, ${ }^{25}$ Japan Atomic Energy Agency (JAEA), Tokai-mura, Japan, ${ }^{26}$ European Commission, Joint Research Centre, Geel, Retieseweg 111, B-2440 Geel, Belgium, ${ }^{27}$ Karlsruhe Institute of Technology, Campus North, IKP, 76021 Karlsruhe, Germany, ${ }^{28}$ National Technical University of Athens, Greece, ${ }^{29}$ School of Physics and Astronomy, University of Edinburgh, United Kingdom, ${ }^{30}$ Istituto Nazionale di Fisica Nucleare, Sezione di Bologna, Italy, ${ }^{31}$ Dipartimento di Fisica e Astronomia, Università di Bologna, Italy, ${ }^{32}$ Istituto Nazionale di Fisica Nucleare, Sezione di Legnaro, Italy, ${ }^{33}$ Consiglio Nazionale delle Ricerche, Bari, Italy, ${ }^{34}$ Istituto Nazionale di Fisica Nucleare, Sezione di Trieste, Italy, ${ }^{35}$ Dipartimento di Fisica e Astronomia, Università di Catania, Italy, ${ }^{36}$ University of Ioannina, Greece, ${ }^{37}$ University of Vienna, Faculty of Physics, Vienna, Austria, ${ }^{38}$ University of Granada, Spain, ${ }^{39}$ Department of Physics, University of Basel, Switzerland, ${ }^{40}$ Centre for Astrophysics Research, University of Hertfordshire, United Kingdom, ${ }^{41}$ Bhabha Atomic Research Centre (BARC), India, ${ }^{42}$ Australian National University, Canberra,

\section{Abstract}

The neutron time of flight (n_TOF) facility at CERN is a spallation source characterized by a white neutron spectrum. The innovative features of the facility, in the two experimental areas, $(20 \mathrm{~m}$ and $185 \mathrm{~m})$, allow for an accurate determination of the neutron cross section for radioactive samples or for isotopes with small neutron capture cross section, of interest for Nuclear Astrophysics. The recent results obtained at n_TOF facility are presented. 


\section{Introduction}

Neutron capture reactions are the main mechanisms for synthesizing elements heavier than $\mathrm{Fe}$ in stars. About half of the elemental abundances are produced by the rapid neutron capture process, or r-process. This process, associated to explosive stellar scenario, is characterized by very high neutron densities, higher than $10^{20} \mathrm{~cm}^{-3}$. In this process the reaction flow is driven towards the neutron rich side, since neutron captures are faster than radioactive decays. The other half of elemental abundances are generated in the slow neutron capture process, the sprocess, in which progressively heavier elements are produced by neutron captures and subsequent $\beta$-decays on seed nuclei as $\mathrm{Fe}$ and $\mathrm{Ni}$. The stellar environment in which s-process takes place is characterized by neutron densities between $10^{6}$ and $10^{12} \mathrm{~cm}^{-3}$. In these sites, the nucleosynthesis path proceeds along the stability valley, since $\beta$-decays are usually faster than subsequent neutron captures on unstable species. Exceptions are the radionuclides with a relatively long half life, where $\beta$-decays and neutron capture may compete. Such nuclei are called branching points and their study can provide important information about the s-process environments.

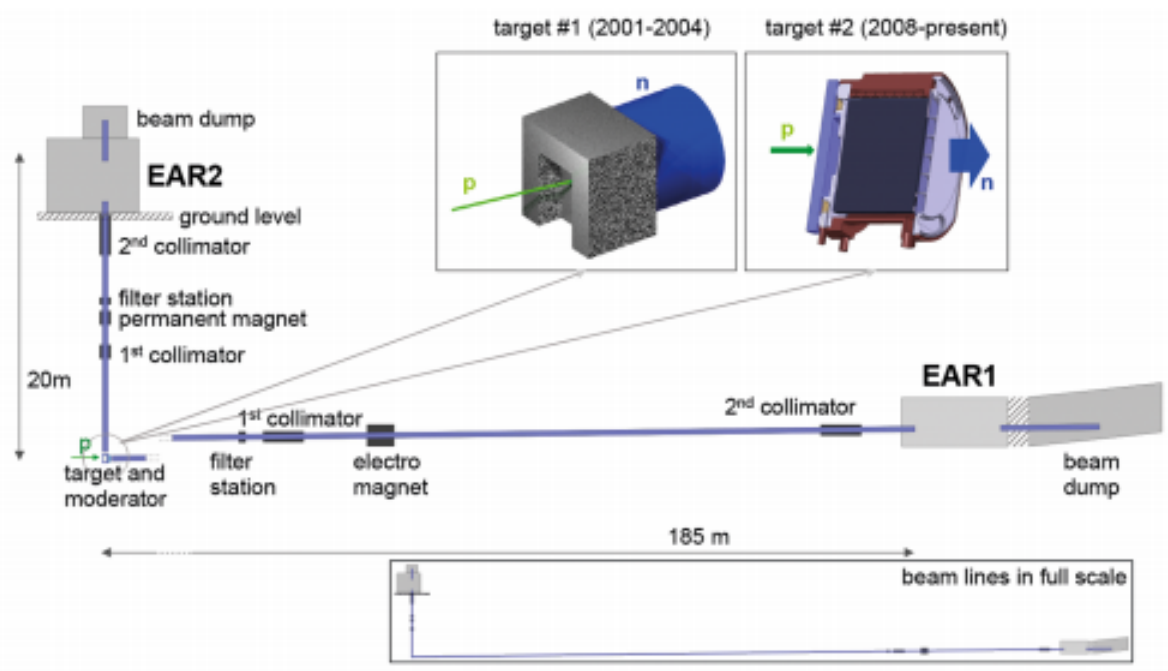

Fig. 1 Sketch of the n_TOF facility beam lines and experimental areas (EARI and EAR(2) and of the to spallation target used up to now. The picture is to scale [3].

\section{The n_TOF facility}

To address the needs of new data of interest for Nuclear Astrophysics, as well as for basic and applied Nuclear Physics, a neutron time of flight facility, n_TOF, was proposed and built at CERN in 2001 [1]. The facility is a pulsed white spectrum neutron source covering a wide neutron energy range [2]. Neutrons are produced in a monolithic $\mathrm{Pb}$-spallation target, where a pulsed $20 \mathrm{GeV} / \mathrm{c}$ proton beam provided by the CERN Proton Synchroton (PS) impinges with a maximum repetition rate of $0.8 \mathrm{~Hz}$. The $\mathrm{Pb}$-target is surrounded by a water layer acting both as coolant and moderator of the neutron spectrum. 
At present two beam lines are in operation, one in the horizontal direction and the other one, recently built, on the vertical of the spallation target. A schematic drawing of the facility is shown in Fig. 1. The two insets depict the two spallation targets used up to now. The neutron flux in the two experimental areas, in units of lethargy, is shown in Figure 2.

The strong suppression of the thermal neutron peak in EAR1 is due to the use of ${ }^{10} \mathrm{~B}$ loaded moderator, whose main purpose is to suppress the $\gamma$-background related to neutron capture in water. The first experimental area (EAR1), operational since the start of the facility, is placed at a distance of approximately $185 \mathrm{~m}$ from the spallation target. More details on this area can be found in Ref. [4].

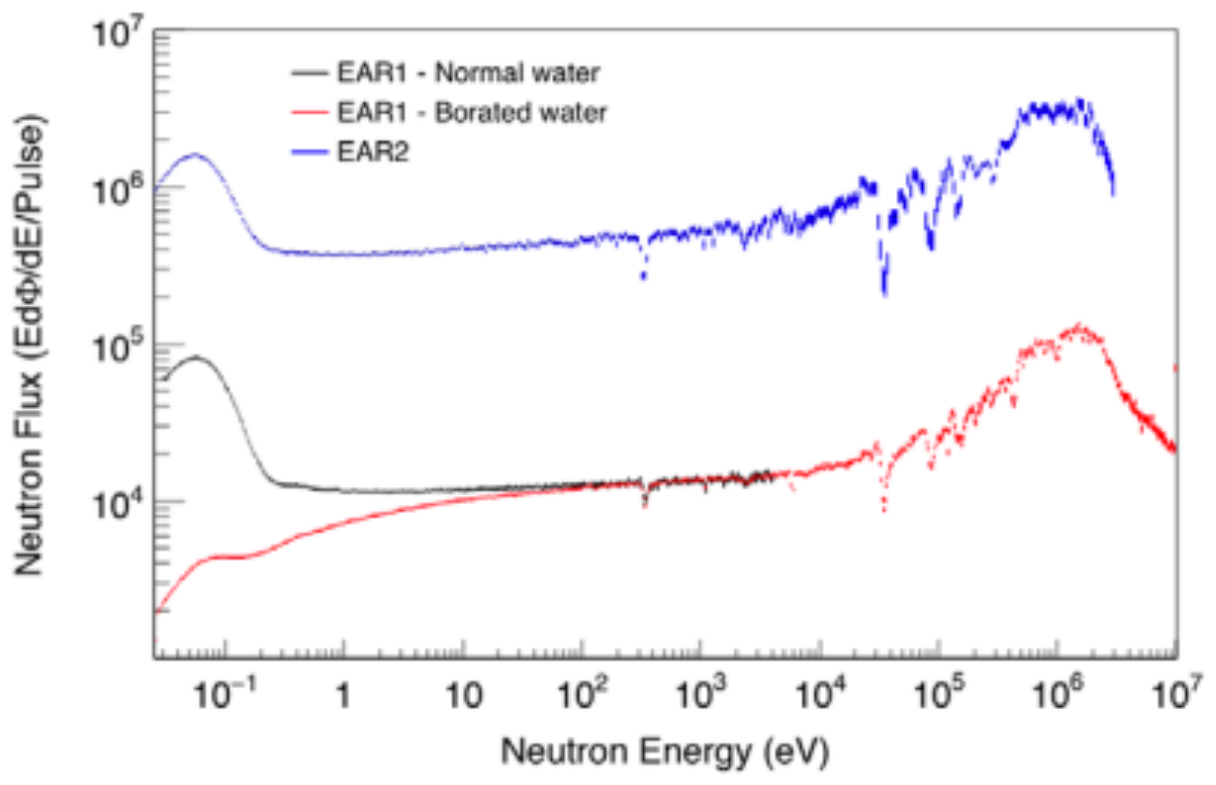

Figure 2 n_TOF neutron flux at EARl with normal (black) and borated (red) water as moderator compared with neutron flux at EAR2 (blue)

The second neutron beam and experimental area (EAR2) has been operational since 2014 . The flight path of this line is approximately $20 \mathrm{~m}$.

The long flight path of EAR1 results in a very high energy resolution, whereas the short flight path of EAR2 leads to a significantly higher neutron flux. The large gain in neutron flux, of about a factor of 25 relative to EAR1, allows one to perform measurements with samples of correspondingly smaller mass or in a shorter time. Most importantly, the combination of the higher flux and shorter time-of-flight, a factor of 10 relative to EAR1, is particularly convenient when measuring radioactive isotopes, as it results in an increase of the signal-to-background ratio of more than two orders of magnitude for the background related to the radioactive decay of the sample. As a consequence, in EAR2 it becomes feasible to perform challenging measurements on isotopes with half-life as short as a few years, offering the unique opportunity to address some open questions in Nuclear Astrophysics. 


\section{Measurements at the n_TOF facility and their implications}

The $n \_$TOF experimental program for Nuclear Astrophysics is mainly focused on neutron magic nuclei, which act as bottle neck for the reaction flow of the s-process, nuclei with $\mathrm{A}<$ 120 , branching poins isotopes and isotopes of special interest, like the Os isotopes relevant for nuclear cosmochronology.

Of particular interest are the branching points, i.e. radioactive isotopes of relatively short half-life for which a competition exists between neutron capture and $\beta$-decay. The neutron capture cross sections of these isotopes are poorly known, manly due to the difficulties in producing samples of sufficient mass and adequate purity, as well as due in handling and measuring samples of very high activity. For these reasons, before 2001 very few measurements existed for these isotopes, at a time-of-flight facility, despite the fact that they could provide very important information on the thermodynamical conditions of the stellar site in which sprocess occurs.

In the following a description of the most recent measurements carried out in the two experimental areas is reported.

\section{1 ${ }^{171} \operatorname{Tm}(\mathrm{n}, \gamma),{ }^{147} \mathrm{Pm}(\mathrm{n}, \gamma)$ and ${ }^{204} \mathrm{Tl}(\mathrm{n}, \gamma)$ measurements}

The isotopes ${ }^{171} \mathrm{Tm},{ }^{147} \mathrm{Pm}$ and the ${ }^{204} \mathrm{Tl}$ are important s-process branching points [5]. The unstable isotope ${ }^{171} \mathrm{Tm}$ (half-life 1.92 years) represents a branching in the s-process path that is independent of stellar temperature and therefore suited to constrain explicitly the s-process neutron density in low mass AGB stars. Being Tm a rare earth element, the relative abundances of stable the isotopes are known with high accuracy.

The isotope ${ }^{204} \mathrm{Tl}$ (half life 3.8 years) decays in ${ }^{204} \mathrm{~Pb}$, which produces ${ }^{205} \mathrm{~Pb}$ when undergoing neutron capture. The fact that both isotopes ${ }^{204,205} \mathrm{~Pb}$ are screened from the r-process by the stable isotopes ${ }^{204} \mathrm{Hg}$ and ${ }^{205} \mathrm{Tl}$, makes ${ }^{204} \mathrm{Tl}$ particularly interesting, indeed, its capture cross section is of crucial importance for understanding the nucleosynthesis of heavy elements in AGB stars, but it can also be used to provide chronometric information about the time span between the last s-process nucleosynthesis events that modified the composition of the protosolar nebula and the formation of solar system solid bodies [6].

The ${ }^{147} \mathrm{Pm}$ isotope is a branching point in the mass region $\mathrm{A}=147-148$, that is the Nd-Pm$\mathrm{Sm}$ region. A detailed analysis of this branching is important for modeling the AGB star evolution and to put accurate constraints on the interplay between metallicity and initial stellar mass, mixing processes or hot bottom burning effects [7].

All these isotopes are radioactive and with a relatively short half life. As a consequence of the natural activity of the sample, the measurement of the neutron capture cross section requires a very large instantaneous neutron flux Anther big challenge is to find a sufficient amount of material with adequate purity.

The samples were all produced at the Institute Laue Langevin ILL (Grenoble, France) high flux reactor, by irradiating for several weeks under the thermal neutron beam the stable progenitors with thermal neutrons. 


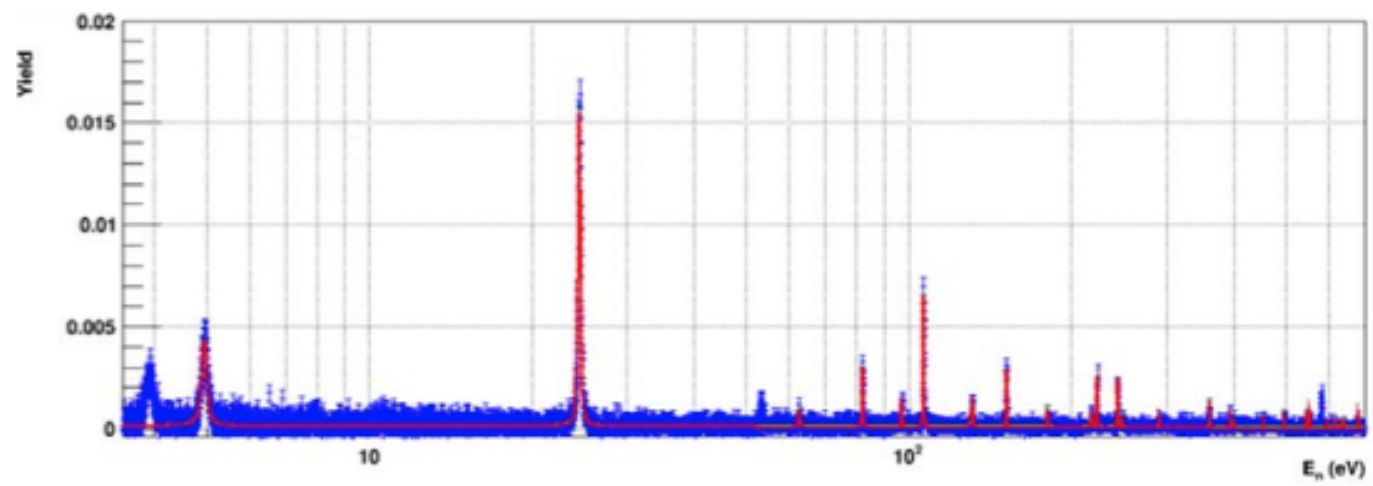

Figure 3 Experimental capture yield of the ${ }^{171} \operatorname{Tm}(n, \gamma)$ measurement [8]

The measurements were performed both in EAR1 and EAR2 with $\mathrm{C}_{6} \mathrm{D}_{6}$ liquid scintillator detectors. The data analysis is currently in progress [8,9], Fig 2 reports the preliminary results of the ${ }^{171} \operatorname{Tm}(\mathrm{n}, \gamma)$. When the analysis will be completed it will provide for the first time experimental information on the capture cross section of these isotopes.

\section{2 $2^{7} \operatorname{Be}(n, \alpha)$ and ${ }^{7} \operatorname{Be}(n, p)$ measurements}

One of most important unsolved problems of nuclear astrophysics is the so called "Cosmological Lithium problem" [10]. It refers to the large discrepancy between the abundances of primordial ${ }^{7} \mathrm{Li}$ predicted by the standard theory of Big Bang Nucleosynthesis $(\mathrm{BBN})$ and the value inferred from the so-called "Spite plateau" in halo stars. The predictions of the BBN theory reproduce successfully the observations of all primordial abundances except for ${ }^{7} \mathrm{Li}$, which is overestimate by more than a factor of 3 .

In the standard theory BBN, 95\% of primordial ${ }^{7} \mathrm{Li}$ is produced by the decay of ${ }^{7} \mathrm{Be}$ $\left(t_{1 / 2}=53.2\right.$ days). Several mechanisms have been put forward to explain the difference between calculations and observation. One possible explanation of the primordial ${ }^{7} \mathrm{Li}$ problem is related to the BBN calculations on the production and destruction of ${ }^{7} \mathrm{Be}$. In particular, while the main reaction producing ${ }^{7} \mathrm{Be}$, the ${ }^{3} \mathrm{He}(\alpha, \gamma)^{7} \mathrm{Be}$ reaction, is relatively well known, the cross section for several reactions responsible for its destruction were still uncertain until recently. To this end several measurements have been performed for charge-particle induced reaction on ${ }^{7} \mathrm{Be}$. The results have ruled out the possibility that reaction induced by proton, deuterons or ${ }^{3} \mathrm{He}$ could be responsible for the destruction of ${ }^{7} \mathrm{Be}$ during $\mathrm{BBN}$.

In the BBN scenario, neutron-induced reactions on ${ }^{7} \mathrm{Be}$ also play a role. However, despite of their importance in the BBN context, very few and uncertain experimental data are available for these reactions. In 1988 the ${ }^{7} \mathrm{Be}(\mathrm{n}, \mathrm{p})$ cross section was measured at the LANCSE neutron facility, Los Alamos. The result excluded a significant impact of this reaction on the ${ }^{7} \mathrm{Li}$ problem [11]. However, because of the limited energy range covered in that measurement, the estimation of the reaction rate at BBN temperature had still to rely on some assumption. Therefore a more precise measurement at temperature between $\mathrm{kT}=25-50 \mathrm{keV}$ is needed to improve the reliability of the BBN calculations. 
The contribution of the ${ }^{7} \mathrm{Be}(\mathrm{n}, \alpha)$ reaction to the destruction of the ${ }^{7} \mathrm{Be}$ has always been considered negligible in the BBN calculation, due to its much lower estimated cross section. However, this assumption has never been verified experimentally, so that an uncertainty of a factor 10 is typically assigned to this reaction in BBN calculation [12].

The lack of experimental data for this reaction is essentially due to the intrinsic difficulty of the measurement, related to the specific activity of ${ }^{7} \mathrm{Be}$ (about $13 \mathrm{GBq} / \mu \mathrm{g}$ ) and the availability of ${ }^{7} \mathrm{Be}$ in sufficient quantity.

The measurement was performed in EAR2. Two samples of $20 \mathrm{GBq}$ each (corresponding to $\sim 2 \mu \mathrm{g}$ ), were prepared at the Paul Scherrer Institute (PSI), Villigen, with ${ }^{7}$ Be extracted from the cooling water of the SINQ spallation source. In the ${ }^{7} \mathrm{Be}(\mathrm{n}, \alpha){ }^{4} \mathrm{He}$ reaction, two $\alpha$-particles are emitted back to back with a relatively high energy of approximately $9 \mathrm{MeV}$. The $\alpha$-particles were detected in two $\mathrm{Si}$-sandwiches-detectors with a sample of ${ }^{7} \mathrm{Bi}$ in between. The two $\alpha-$ particles were identified on the basis of their relatively high energy and by the coincident method.

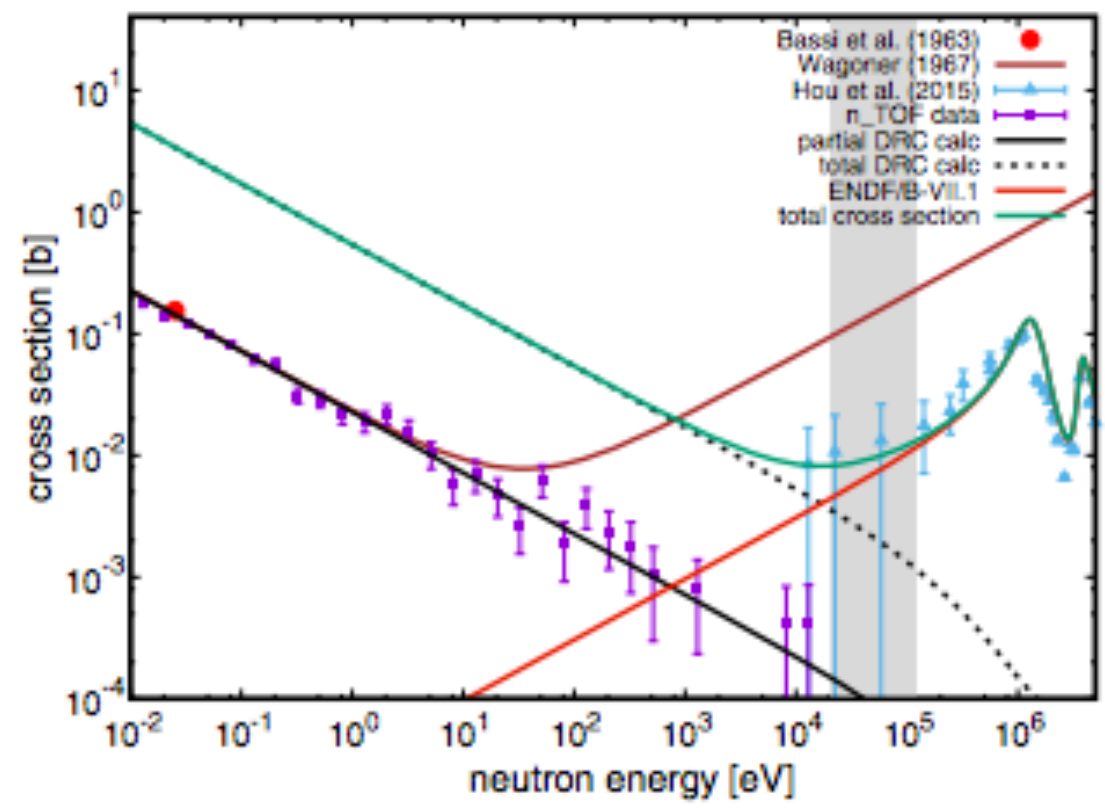

Figure 4 The partial ${ }^{7} \mathrm{Be}(n, a)$ cross section measure at $n \_$TOF [13] compared with estimated cross section and the previous measurement at thermal energy [14]

The energy-dependent cross section of this reaction was measured for the first time in a wide range of neutron energies, from thermal to $10 \mathrm{keV}$. More details on the results can be found in Ref. [13]. Figure 4 shows the partial ${ }^{7} \operatorname{Be}(n, \alpha)$ cross section compared with previous cross section data used for the BBN calculation.

The present results are in good agreement at thermal neutron energy with the only previous measurement [14]. The total cross section has been estimated on the basis of the measured partial cross section and theoretical calculations of the Direct Radiative Capture (DRC) mechanism. The estimated total cross section is almost a factor of 50 higher than 
previously thought at low energy, but it keeps decreasing at higher energy, being a factor of 10 lower than previous evaluations in the range of interest for BBN. Although these results clearly show the inadequacy of the cross section estimates used so far for this reaction in BBN calculations, they unambiguously indicate that this reaction cannot solve the Cosmological Lithium Problem, whose solution should then be searched for in alternative physical scenarios.

\section{Conclusion}

Since 2001 the $\mathrm{n}_{-}$TOF facility at CERN has been providing valuable data on neutron capture reactions of interest for nuclear astrophysics, as well as for energy and medical applications. The unique features of high flux and resolution have allowed in many cases to reduce the uncertainties on cross sections of various isotopes involved in s-process nucleosynthesis. In particular, high accuracy data have been collected on radioactive nuclides around branching points, on bottleneck magic nuclei, or on isotopes available only in small amount, of the order of a few milligrams. The construction of a second experimental area at a shorter flight path has recently opened the way to even more challenging measurements of $(n, \gamma)$ and (n,charged particle) reaction on isotopes of short half-life, low cross section and limited amounts (possibly down to a few micrograms). All this is expected to lead in the near future to exciting new results and significant advancements towards the refinement of models of stellar nucleosynthesis.

\section{References}

[1] C. Rubbia et al, Tech Rep. CERN/LHAC/98-02 CERN (1998)

[2] U. Abbondanno et al., Trch. Rep. CERN/SL/2002-053 ECT (2003)

[3] F. Gunsing et al., Eur. Phys. J. Plus 131, 371 (2016)

[4] C. Guerreo et al., Eur. Phys J. A 49, 27 (2013)

[5] F. Käppeler et al., Rev. Mod. Phys 83(2011)

[6] K. Yokoi, K. Takahashi and M. Amould, Astronomy and Astropysics 145(1985) 339

[7] F. Herwig, Anny. Rev. Astron. Astrophys. 43, (2005) 435

[8] C. Guerrero et al., Jap. Phys. Soc. Conference proceeding, accepted

[9] C. Guerrero et al., Eur. Phys. J. web of conference, accepted

[10] R.H. Cyburt et al., Phys. Rev. D 69123519 (2004)

[11] P. Koehler et al., Phys Rev. C 37, 917 (1988)

[12] P.D. Serpico et al., Jour. Cos. Astropart. Phys 12, 010 (1994)

[13] M. Barbagallo et al., Phys. Rev. Let. 117, 152701 (2016)

[14] P. Bassi et al., Il Nuovo Cimento XXVIII, 1049 (1963) 